\title{
Cooperative Image Workstation Based on Explicit Models of Diagnostic Information Requirements
}

\author{
Thomas Wendler, Rüdiger Grewer, Karl J. Mönnich, Joachim Schmidt, and Heino Svensson
}

\begin{abstract}
A medical image workstation designed to act as a cooperative dialogue partner in diagnostic radiology has been conceived, and a prototype has been made. The system can automatically select relevant information (eg, from current and previous examinations) and generate a meaningful and appropriate image arrangement on the display screen. For a number of routinely performed tasks in radiology, the users' interaction may be as simple as switching from one patient to the next. This is shown to considerably simplify and speed up radiological image access and presentation, saving the user time and effort. The cooperative system response is based on explicit (formalized and computeraccessible) models of diagnostic information requirements. These models are context dependent and take into account that diagnostic information needs vary with radiological work procedures, workstation users, and patient cases. Initial models have been acquired from expert radiologists in two European hospitals and were integrated in a cooperative workstation prototype. For the representation of models, rulebased and object-oriented techniques were applied. The rule base was designed with a distinct modular structure, separating between rule sets for general, task-dependent, and user-dependent information requirements. The installed rule-based mechanism also offers a solution for the automatic prefetching of images to avoid transmission delays in the course of diagnostic work sessions. The first part of the report reviews the objectives for the design of cooperative workstation user interfaces and explains the benefits from the users' point of view. In the second part, the acquisition, structuring, formalization, and representation of context-dependent information requirement models is described. The rule-based model is explained using examples. A layered workstation architecture consisting of model, object, and real-time layers is presented. Difficulties in the implementation of cooperative workstations are discussed that point to future research topics and standardization efforts.
\end{abstract}

From the Department of Technical Systems, Philips Research Laboratories, Hamburg, Germany.

The work described here was part of the HIPACS project ${ }^{7}$ funded by the European Community under grant AIM (Advanced Informatics in Medicine) $A 1008$ and is now funded as part of the EuriPACS project under grant A2009.

Address reprint requests to Thomas Wendler, PhD, Philips GmbH Forschungslaboratorien, Dept Technical Systems, Hamburg, PO Box 540840, D-2000 Hamburg 54, Germany.

Reprinted with permission from Medical Imaging VI: Picture Archiving and Communications Systems, Society of PhotoOptical Instrumentation Engineers, Vol 1653, 1992.

Copyright 11992 by The Society of Photo-Optical Instrumentation Engineers

0-8194-0805-0/92/\$4.00
KEY WORDS: image workstation, cooperative user interface, information requirement model, knowledgebased system, knowledge acquisition, object-oriented design, rule-based model.

$A$ S DIGITAL information systems in diagnostic radiology become more complex and more widely used, there is a strong demand to increase their usability by appropriate user interface design. In the discussion of userfriendly image workstations, "simplicity" and "ease of use" are frequently mentioned keywords. To fulfill these requirements, the user interface approach presented in this report was taken ultimately to simplify and speed up image access and presentation in various diagnostic work settings.

It is well known that systems cannot be user-friendly in general, but only with respect to specific users and work settings. In human factors research, it is agreed that appropriate user interfaces can be approached by a predominantly user-centered design process, such as that proposed by Norman. ${ }^{1}$ However, usercentered design techniques often result in compromises that focus on the average user and assume a number of general goals that users may be interested in. Changing requirements that develop from different users and the diversity of goal-oriented tasks and subtasks are rarely considered during system design and almost never considered at system run time. Most human-machine interfaces seem to be static: they function uniformly, regardless of what kind of user is working with a system and what actual task the user is concerned with.

Cooperative user interfaces, in contrast, conceptually take into account that workstation users and their actual tasks, and thus their current goals and information requirements, are variable at run time. In radiology, digital image workstations will be used by quite different stereotypic categories of users (eg, principal or assistant radiologists, physicists, technicians) with diverging goals, responsibilities, and information requirements. Even within a particular user group, personal preferences and styles are observed (eg, how images should be arranged 
on the display screen). Analyzing the film-based diagnostic process, various categories of goaloriented tasks can be distinguished, ranging from simple control actions (eg, image quality check after the acquisition) to quite complex image handling and display procedures (eg, preparing a demonstration session for referring clinicians). A variation of information requirements is obvious for different tasks.

Furthermore, with the introduction of digital technologies and advanced system features, initial user requirements can be expected to change. Therefore, an easy adaptation of user interfaces to developing user and task requirements will be particularly helpful in the introduction phase of fully digital information systems in radiology, in which new image handling and viewing procedures will probably develop.

Based on these general assumptions, an image workstation was designed that can automatically select and present multimodality images in a cooperative manner based on contextdependent, explicit models of information needs. The overall design objectives can be summarized as follows: (1) to increase the efficiency of routinely performed work procedures by simplifying the users' interaction and speeding up information access and presentation; (2) to increase the effectiveness and accuracy of the diagnostic work by volunteering needed information (presenting all information that contributes to a problem) and by focusing on relevant information (discarding the vast amount of irrelevant information); and (3) to increase the usability and acceptability of digital information systems in radiology by simple, convenient, and fast information access and problem-fitting information presentation.

From the user's point of view, cooperative actions should be adequate and supportive. They must not result in surprising and disturbing effects (the underlying models for cooperative actions must be correct). There is also a strong demand that consistency and predictability of user interfaces are not affected by the adaptation mechanisms. An adequately designed static user interface is an important precondition for a successful cooperative system.

From the workstation designer's point of view, part of the workstation's functionality must be controlled by explicit, formalized, and computable descriptions (models) of contextdependent diagnostic information requirements. These models not only will guide the design process (eg, as suggested by Braudes ${ }^{2}$ ) but will define distinct parts of the workstation's functionality at run time. This allows for interactive modifications and adaptations of the functionality on a high level of abstraction (in our case expressed by rules ${ }^{3}$ ). Because the information requirement models must be acquired in close collaboration with the user community, a user-centered design concept is mandatory for cooperative user interfaces.

Following this general strategy, a cooperative diagnostic image workstation has been conceptualized and the underlying models of information needs have been acquired and implemented. In the discussion of these achievements, the following definitions of terms apply: (1) diagnostic context: a diagnostic work setting characterized by the current workstation user, diagnostic task, and patient case; (2) diagnostic information requirements: all material (documents, facts, data, knowledge) needed in a diagnostic context for successful diagnostic work; (3) adaptive workstation user interface: a user interface that changes a distinct part of its functionality at system run time, if a specific diagnostic context is presumed; (4) cooperative workstation user interface: an adaptive workstation user interface that actively supports users in reaching their goals in the diagnostic process by providing relevant information in a given context (on self-initiative or on request). Cooperative actions are based on explicit models of context-dependent diagnostic information requirements.

\section{THE COOPERATIVE WORKSTATION USER INTERFACE}

From the many possible ways that a cooperative user interface could be supportive in diagnostic radiology, ${ }^{4}$ the following principal mechanism was selected and implemented. The workstation was designed to volunteer (automatically select, gain access to, and display) needed pieces of information in a given diagnostic context. Thus, the system should be seen by its users as a cooperative dialogue partner that actively (on self-initiative, eg, after patient selec- 
tion) or passively (on explicit request) attempts to fulfill the user's current diagnostic information requirements. Practically, the scope of the mechanism described in this report was restricted to the cooperative selection and display arrangement of image-type documents (images and image sequences).

The effect of cooperative actions may be best shown by examples of different options for the degree of self-initiative. The three examples in Fig 1 present a typical sequence of image access and display operations that may occur in a routine reporting session. The actions taken by the user and the workstation are shown separately on both sides on the drawings. The example session consists of the following: session start (user $\log$ in procedure and task definition), selection of the first patient from a list of patients, selection of the patient's relevant images from a pictorial index, image access and image arrangement on the display screen, image reading, report dictation, and next patient(s).

Figure 1A shows a possible sequence of actions with a noncooperative, static user interface. The interaction for selecting images and arranging them on the display screen may be based on direct manipulation techniques with pointing devices, as typical for most state-of-theart user interfaces. The list of patients and the pictorial index of images show all current patients and the complete content of their image files. An example of the designed workstation's image screen with an opened pictorial index folder is shown in Fig 2. The arrangement of images on the viewing area follows fixed mechanisms, eg, images selected from the folder appear in adjacent viewports with reduced size or in overlapping viewpoints with the best possible resolution in the middle of the screen.

Figure 1B shows a possible sequence of actions with a cooperative user interface (option 1). In this case, only a selected subset of patients (relevant for this radiologist and his/her current task) is listed and only relevant images appear as minified versions in the pictorial index (remaining nonrelevant examinations may be presented as text string). As a typical example, from 80 images found in a patient file only 2 "mandatory" images (from the current examination) and 4 images "of additional interest" (from previous examinations) would be pre-

USER

WORKSTATION

USER

WORKSTATION

USER

WORKSTATION

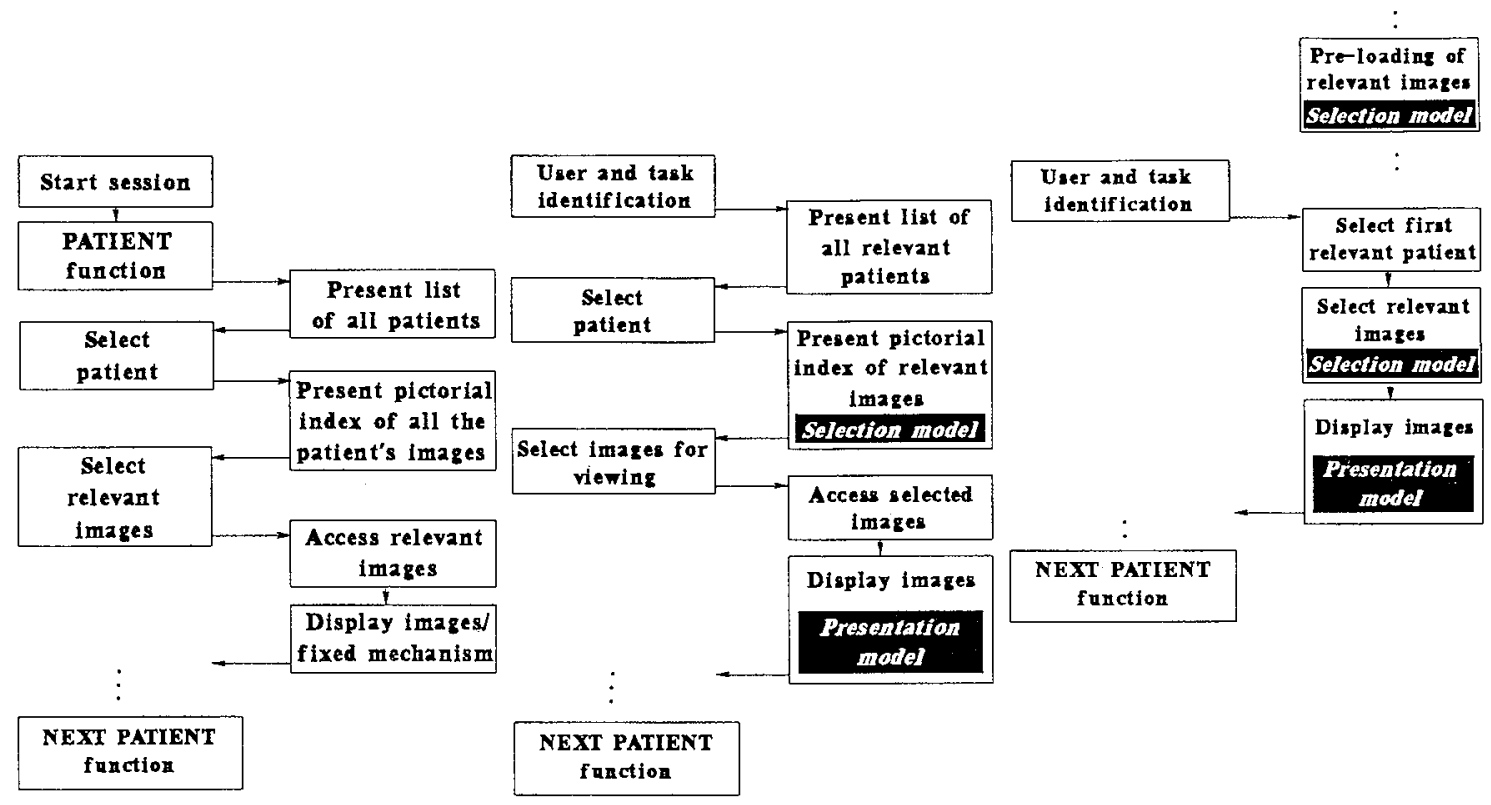

A

B

C

Fig 1. Example session with static user interface (A) and with different options for cooperative user interfaces (B and $C)$. 
Fig 2. Part of the workstation's image screen with opened pictorial index folder.

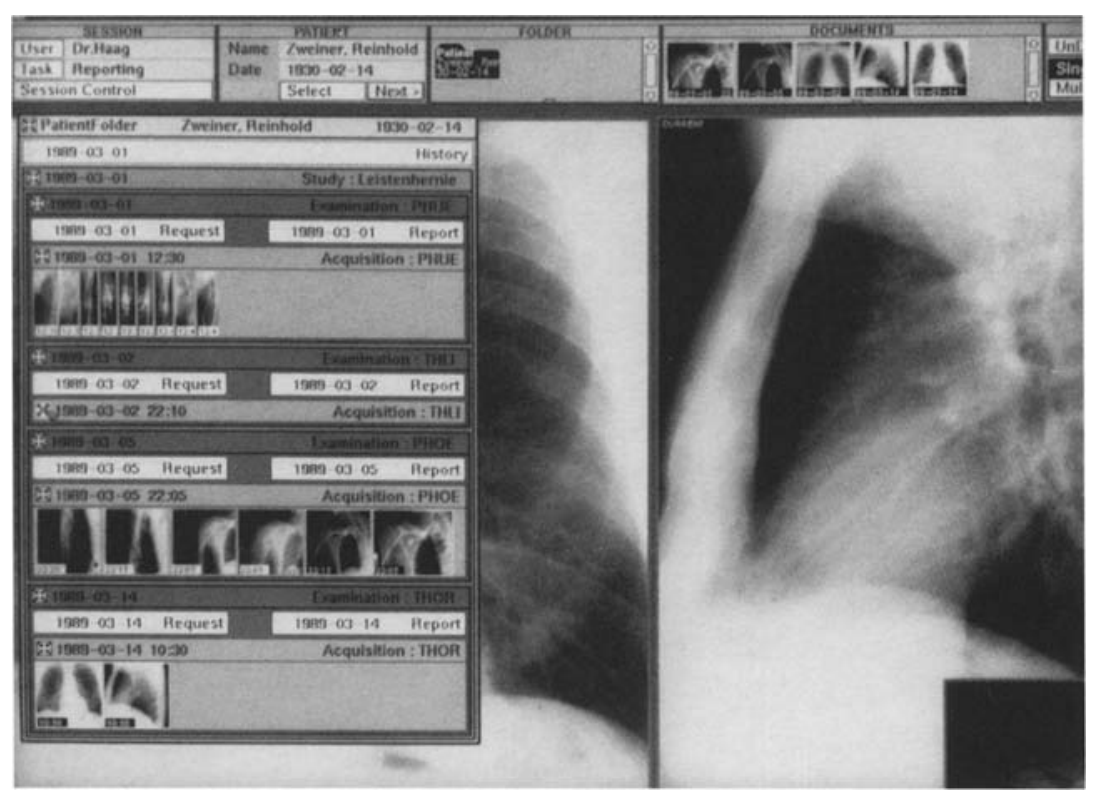

sented. The terms mandatory and of additional interest refer to an image-classification scheme described later in this report. The image arrangement (after selection) is controlled by a presentation model. In option 1, the selection process from lists and pictorial indexes is left to the user.

Figure $1 \mathrm{C}$ gives an alternative sequence of cooperative actions that shows the far more extended scenario (option 2) that was finally implemented in the workstation prototype. Before the users' work sessions, images expected to be relevant for the anticipated tasks are selected and preloaded into the workstation's fast-access memory. During the session, the selection process from lists and pictorial indexes is automated. In principle, this mechanism generates (immediately after user identification and task identification) an appropriate presentation of all relevant images for the first relevant patient. Later, the session may continue by simply activating a "NEXT PATIENT" menu field. Selection criteria for preloading must consider all anticipated tasks and users and therefore will generally differ from those used in specific work settings.

A cooperative user interface such as this can significantly simplify and speed up image access and presentation. For many tasks in diagnostic radiology, the users' interaction may be as simple as switching from one patient to the next.
From the radiologists' point of view, this interface can save considerable time and effort. If appropriate, images from previous studies will automatically be shown for comparisons without time-consuming searches in patient folders. The image arrangement may be tailored to the users' needs or preferences. Despite the system's cooperative actions, the user may at any time take the initiative to perform other tasks if required, eg, look at the complete list of patients, look at the complete content of the patient's image folder, select additional images, or rearrange the images on the screen.

\section{EXPLICIT MODELS OF DIAGNOSTIC INFORMATION REQUIREMENTS}

After discussing some of the features and benefits of cooperative user interfaces, the next paragraphs will describe the technical approach taken for the realization of a workstation prototype. The cooperative user interface is based on formal descriptions of context-dependent diagnostic information needs. These explicit models have been acquired in close collaboration with radiologists from two European hospitals (The University Hospital of Aachen [Clinic for Diagnostic Radiology] and the University Hospital of Brussels [AZ-VUB, Department of Radiology]). In the following, the acquisition, structuring, formalization, and representation of models will be explained. 


\section{Methods for Knowledge Acquisition}

To acquire and formulate models of diagnostic information requirements, investigations were set up to clarify the following questions. (1) Which documents (especially images and image sequences) from previous examinations are relevant in the context of the current examination, and which properties (document parameters) define the criteria that make them relevant? (2) How should current and previous images be arranged on the display screen? and (3) How can the acquired knowledge of diagnostic information requirements be structured, formalized, and implemented in a computer executable representation system? A general objective for modeling image selection and arrangement was to focus, whenever possible, on the goals of the diagnostic process rather than on the features and limitations of currently used tools (film folder, lightboxes, etc). For the investigations, the following methods were used.

Introduction to department organization and data flow. In a number of meetings, a general introduction to the radiological procedures and a detailed explanation of department organization and image data flow were given in both hospitals by members of the clinical staff. In addition, typical goal-oriented radiological work procedures were defined. The staff involved in radiology was described in terms of their roles in the diagnostic process.

Inventory and classification of all documents and data of radiological interest. A complete list of all routinely used documents (eg, images, image sequences, reports, request forms) and document collections (eg, various kind of folders, acquisitions, examinations, studies) was assembled. All document types were described in terms of their typical properties and their relation to each other. A common terminology was developed. A list of all routinely performed examination types was provided by the University Hospital of Aachen (a total of 386 different types of examinations), and a hierarchical anatomic description was designed and assigned to each examination type. In the course of the project, the assembled information on the documents of radiological interest was directly translated into an object-oriented representation system.
Interviews with radiologists (recorded on audio tape). In the search for heuristics of diagnostic information requirements, principal radiologists and radiological assistants in both hospitals were interviewed. The participants were encouraged to formulate their answers in terms of rules. In Aachen, the interviews were recorded on audio tape and the written results of interviews were iterated in various subsequent sessions. After the knowledge was structured and formalized in a rule-based system, interviews were also used as an initial step to validate the acquired knowledge. Interview questions focused on the relevance of various document types in different work situations. Radiologists were asked to define information requirements that arise, eg, from a given diagnostic task, clinical question, current examination type, or the patient's actual disease.

Protocols of routine viewing sessions (using questionaires). Protocols of real viewing sessions were taken at varying work places in the radiology department to detect observable criteria for the selection of relevant images and their display arrangement. To support this, a questionnaire was designed to capture the relevant parameter sets during the work sessions. It seemed desirable to have complete protocols of the currently used (displayed) images and additionally of all available documents belonging to a particular patient (even those that existed but were not available because of loss). The image arrangement was also sketched on the questionaire. During the sessions, radiologists were asked to assess the image arrangement using five categories: (1) mandatory from the medical point of view, (2) appropriate (but not critical), (3) accepted clinical standard (may vary among hospitals or departments), (4) personal style (subjective preference), and (5) pragmatic reasons (eg, limited space on lightbox). Although this was a promising approach to objective detection of information requirements, it was difficult to complete the entire questionnaire in synchronism with the radiologists' working speed. Altogether, the reading of 108 examinations was observed in reporting sessions at different work places in the department.

Simulated viewing sessions with selected cases. Simulated clinical viewing sessions were carried out with well-prepared and completely known 
patient cases mainly to validate results of the initial knowledge acquisition. As an advantage of this method, all image material and clinical information of a patient were available (complete image folder and all alphanumeric documents). In a simulated environment it was possible for the investigator to ask the radiologists for deeper explanations. In the University Hospital of Aachen, 10 representative cases were selected and discussed in simulated sessions.

\section{Sources of Evidence for the Decision Process}

From clinical knowledge acquisition it became clear that the proposed cooperative actions can be realized only if sufficient sources of evidence (knowledge, facts, data) for the decision process are available. Four principal information sources can be identified.

1. The general domain model of the medical diagnostic process includes knowledge about general procedures and concepts found in radiological practice, eg, heuristics about information requirements arising from categories of diagnostic work such as routine diagnostic, or follow-up diagnostic. Basic knowledge about standard examination types or anatomic and pathological codes also must be available.

2. Task and user models define heuristics about information requirements for specific, predefined diagnostic tasks (eg, reporting, demonstration, image quality check) and for specific user groups (eg, radiologists, technicians) or individuals.

3. Domain status data describe the properties of all currently available radiological objects, eg, all available information on currently existing patients, examinations, images, or diagnostic reports. For cooperative selection processes, the objects of radiological interest must possess a rich set of descriptions from which conclusions can be derived. A typical set of object descriptions is described later in this report. The properties of the available radiological objects are important facts to be used as a basis for cooperative actions.

4. Interactive data were obtained from the user's input during the work session. Useful dialogue data (function calls, object rearrangements, etc) may be gathered and interpreted. The system may also on selfinitiative enter metadialogues to clarify the user's goals or needs, eg, for the current task. In the current implementation, however, the interpretation of interactive data was not considered.

\section{Methods for Knowledge Representation}

In parallel to the clinical knowledge-acquisition activities, efforts were made to structure and formalize the results and find an appropriate knowledge-representation scheme. Two parallel but interdependent modeling approaches were taken.

1. All entities found in clinical radiology (eg, departments, documents, tasks, persons, data) and their properties and relationships were described formally. This can be seen as a descriptive static model of the domain of radiology. Its representation was achieved through an object-oriented system using a language that supports object-oriented design (OBJECTIVE C).

2. The formal description of context-dependent diagnostic information requirements was achieved using a rule-based representation system. In this scheme, models are defined by rule sets that can be activated separately. The various models were implemented using the expert system shell NEXPERT OBJECT.

\section{Object-Oriented Model of the Entities of Radiological Interest}

A complex world of objects found in both of the radiology departments we investigated appears typical for most hospitals. For the implementation of objects, the principles of objectoriented design were applied. In object-oriented systems, all relevant real-world entities are described as strictly encapsulated units (objects) that communicate with each other via messages. Procedures activated in an object by messages are referred to as methods. The objects' methods define the functionality of a static workstationuser interface. A comprehensive introduction to the concepts of object-oriented programming is given by Cox..$^{5}$ An explanation of some of its benefits for the design of image workstations in radiology can be found in Grewer et al. ${ }^{6}$ 
It is useful to structure objects into class hierarchies. Classes are prototypes for object instances, in which instances receive the properties of their class and all super classes (inheritance). Figure 3 shows a fraction of the obtained class structure for some documents of radiological interest. Two kinds of relationships between objects are considered in the diagram: "IS_A" relations (class structure), indicated by solid lines (eg, an MR_IMAGE is a kind of IMAGE, an IMAGE is a kind of DOCUMENT) and "HAS" relations, indicated by dashed lines (eg, an EXAMINATION consists of [= has] a number of acquisitions, an IMAGE SEQUENCE consists of IMAGES). During system operation, "has" relations point to object instances. Only a few example relations are drawn in Fig 3.

Most of these object classes possess a rich set of properties. Taking the example of the object class REQUEST_FORM (in traditional radiology this is a paper form used by referring physician to request a radiological examination), the relevant object properties in Table 1 were found.

Some relevant properties for other objects are shown in Fig 3. As the property values of object instances represent facts that can be tested in the condition parts of rules, they are of central importance for the formulation of cooperative decision processes. In some cases, the allowed values of object properties had to be strictly standardized. For example, clinical questions on request forms are usually "wild" text strings that are hard to interpret with a computer program. To make them available for the decision process, they were divided into a formal "clinical question category" and a free text "clinical question specific part." Mainly the clinical question categories such as "suspicion of. ..," "search for...," "exclusion of...," "condition after...," or "control of. .." were standardized and used in rule conditions. In a

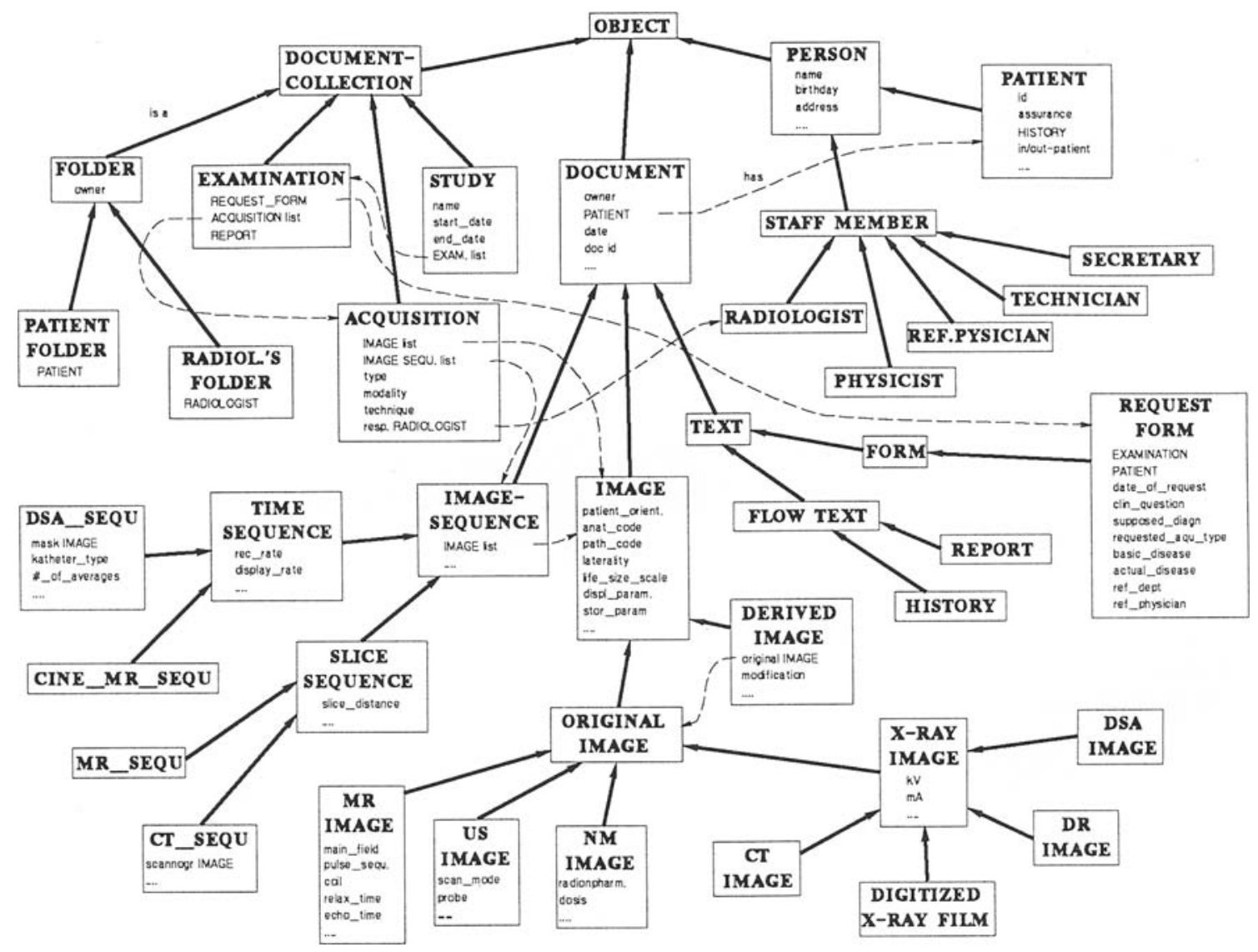

Fig 3. Part of the world of objects found in diagnostic radiology. 
Table 1. Properties of Object REQUEST_FORM

Date of request

Clinical question

Requested examination type

Actual disease

Referring physician

Patient Demographic data (name, birthday, etc)

Supposed diagnosis

Basic disease

Referring department

future request form (being on paper or in a digital information system), the categories can be input by the requester using tick boxes.

\section{Rule-Based Models of Diagnostic Information Requirements}

The information-requirement models will be described using some examples. Participants were asked in interviews to formulate rulelike sentences for the relevance of images and their display arrangement in the context of different diagnostic tasks and patient cases. A large number of rules and other sentences were recorded on audio-tape. Early on it became apparent that the majority of rules focussed on a relatively small set of hypotheses. Typical output of the initial knowledge acquisition phase were statements such as

"If an examination [in a reporting session] can be classified as 'Routine diagnostic,' then only the current images are relevant and images from previous examinations are not relevant. However, dependent on the diagnostic findings, previous images may in some cases become relevant. 'Routine diagnostic' then may change to 'Primary diagnostic'....'

When a definition of the terms used was requested, "routine diagnostic" was defined as an event in which the clinical question associated with an examination is missing or cannot be clarified by the requested examination type (apparent for thorax examinations with typical clinical questions such as "preparation of treatment," "control before blood donation," etc). Furthermore, only thorax examinations were classified as "routine diagnostic." Ten types of conventional thorax examinations were performed in the department (Table 2).

Only the first four examination types (THOR, THBE, THLI, THPA) were assigned "routine thorax candidates." From this point it was feasible to create a number of simple rules that could prove the hypothesis "Is routine diagnostic" and consequently "No previous images relevant." For example,

HYPOTHESIS is_routine_diagnostic

IF

$$
\begin{aligned}
& \text { current_exam.type }=\text { routine } \\
& \text { thorax candidate }(=\text { THOR, } \\
& \text { THBE, THLI, THPA) }
\end{aligned}
$$

AND clinical_question_category = "preparation_of. ..."

Once the decision for a rule-based system had been settled, the main activity was to structure the acquired knowledge and to reformulate and formalize it in terms of valid rules. A set of relevant hypotheses had to be defined with appropriate conditions that could be verified by the system at run time, using facts (properties of object instances) found in the object world. In creating problem-fitting hypotheses, the selection of relevant documents from previous examinations was strongly determined by a construct called "diagnostic category." The diagnostic categories in Table 3 were defined and formulated as rule hypotheses.

For typical pairs (diagnostic category; task), the relevance of previous images was defined using the classification scheme "mandatory," "useful," and "of secondary interest." For example, the transcript of the rule

\section{HYPOTHESIS images_mandatory}

IF

$$
\text { task }=\text { "pre_examina- }
$$$$
\text { tion_viewing" }
$$

AND

$$
\text { diagnostic_category }=\text { "suc- }
$$
ceeding_diagnostic"

AND current_exam.type $=$ "MYE" (myelography)

Table 2. Ten Types of Conventional Thorax Examinations

THOR = thorax in 2 projections

$\mathrm{THLI}=$ thorax with patient lying

THIE = thorax in expiration

THHA = thorax with patient hanging (pediatrics)

THKN = bony thorax

THBE $=$ thorax in bed

THPA $=$ thorax PA projection

THDL $=$ thorax fluoroscopy

THIN = thorax made at the ICU

$\mathrm{KATH}=$ thorax catheter control 
Table 3. Diagnostic Categories Defined by the General Model of Radiology

\begin{tabular}{lc}
\hline Diagnostic Category (Rule Hypothesis) & Description \\
\hline Routine Diagnostic (screening) & clinical questions, mostly in the context of precautions. \\
image interpretation in the context of primary clinical questions (eg, reason for & $\ldots$, suspicion of . . , exclusion of. .). \\
Initial diagnostic & Initial step of primary diagnostic (no radiological history available for the current \\
clinical question). & Subsequent (2nd to $n$ th) step of primary diagnostic (proceeding to more invasive \\
Succeeding diagnostic & and costly types of examinations). A radiological history exists for the current \\
clinical question. & Primary diagnostic under the condition that the same examination type is re- \\
Repeating diagnostic & peated. \\
Follow-up diagnostic & Fontrol actions in the context of a radiological or clinical patient history. \\
Status control & position control of catheter). \\
Collow-up image interpretation that checks for changes by comparison with previ- & ous documents (eg, images before and after treatment, evaluation of the course \\
& of a disease).
\end{tabular}

THEN

mandatory: exam.type $=$ "LWS" (lumbar spine) qualifier: "most recent"

explains that in a pre-examination viewing (in this case, preceding a myelography), the most recently made conventional radiographic images of the lumbar spine in two projections are relevant. These images are known to exist, because myelography is always a succeeding diagnostic step following conventional radiographic images of the spine.

Using a rule-based representation system, models of context-dependent information requirements can be realized as rule sets in which the appropriate set is activated if there is evidence for a given context. To resolve conflicts, models were assigned priorities in which higher-priority numbers overrule lower ones as indicated in Table 4. In this priority scheme, preferences of individual users will overrule, for example, default assumptions defined for a user group or for radiology in general.

User models are activated upon user identification, eg, by log-in procedure. Task models may be activated by declaration (eg, the user may declare a "reporting" task by menu selection) or by default assumptions generated by inference processes (eg, the assumption that this user normally starts reporting at approximately 2 PM).

The task and user models contain hypotheses that define the relevance of images. If the models are used as a consultant in a cooperative user interface, the action parts of the rules may contain varying recommendations on how to react. Examples of hypotheses and the resulting actions implemented are given in Table 5.

Information classified as "useful" or "of secondary interest" are, for example, images from

Table 4. Priority Scheme for Diagnostic Information Requirement Models

\begin{tabular}{lll}
\hline Priority & \multicolumn{1}{c}{ Model Type } & Descriptions \\
\hline 1 & General model of radiology & $\begin{array}{c}\text { Rule set for general information requirements (eg, the definition of } \\
\text { diagnostic categories) and generic object classes of diagnostic } \\
\text { relevance. This rule set will always be activated. } \\
\text { Rule sets for task-specific information requirements. One set at a } \\
\text { time will be activated if there is evidence for a specific task. } \\
\text { Rule sets for user-group specific information requirements (eg, for } \\
\text { radiologists). One set at a time will be activated for a specific } \\
\text { user of that group. } \\
\text { Rule sets for specific information requirements of individuals (per- } \\
\text { sonal preferences). One set at a time will be activated for the } \\
\text { specific individual. }\end{array}$ \\
\hline
\end{tabular}


Table 5. Hypothesis and Possible Actions

\begin{tabular}{|c|c|}
\hline Hypothesis & Adaptation Mechanism (Possible Action) \\
\hline Images mandatory & $\begin{array}{l}\text { Images are preloaded to the workstation's local workfile. After session start and } \\
\text { task selection, these images are displayed automatically. }\end{array}$ \\
\hline Images useful & Images are preloaded but shown only on the user's request. \\
\hline Images of secondary interest & $\begin{array}{l}\text { Images are not preloaded but are shown as miniature versions in pictorial in- } \\
\text { dexes. }\end{array}$ \\
\hline Remaining (irrelevant) images & $\begin{array}{l}\text { Images are not preloaded and are shown in folder indexes as text strings (they } \\
\text { are available only on the user's explicit access request). }\end{array}$ \\
\hline
\end{tabular}

previous examinations that share a common anatomic code with the current examination. For this kind of similarity retrieval, a data base was set up that defines "useful" and "interesting" images by means of a hierarchical anatomic key. The following example shows the codification system:

$\begin{array}{llll} & \begin{array}{l}\text { Anat. } \\ \text { Exam }\end{array} & \begin{array}{l}\text { Sub- } \\ \text { keys }\end{array} & \text { Related exams } \\ \text { THOR } & 630 & 620 & \text { THKN (bony thorax) } \\ \text { (thorax) } & & 321 & \text { BWS (thoracic spine) } \\ & & 308 & \begin{array}{c}\text { LWS (upper lumbar } \\ \text { spine) }\end{array}\end{array}$

Some common patterns were found that define individual preferences for the display arrangement of images or images series. Typical viewing recommendations for multiple or single images (still reflecting film-based viewing) are, for example, parallel viewing, chronological arrangement $(\mathrm{A}, \mathrm{B}, \mathrm{C} \ldots$ left to right/top to bottom); parallel viewing, anatomic arrangement (A, B, C . . . left to right/top to bottom); parallel viewing, paired arrangement (A left of/right of $\mathrm{B}$ ); concurrent viewing in sequence (A, B, C, . .); or image upside down.

An example may show an image arrangement scheme found for two consecutive thorax examinations (current and old examinations, each in posterior-anterior and lateral projections). For comparison in follow-up diagnostic examinations, one preferred display arrangements for these four images was the following:

[(THOR/lat,old)right_of (THOR/pa,old/right_of [(THOR/pa,current)right_of (THOR/lat, current)].

The rule-based models of diagnostic information requirements have been implemented using the expert system shell NEXPERT OBJECT, which offers a convenient graphic user interface for rule browsing and editing. Rules can be easily displayed, modified, and extended. The system offers a variety of inference strategies, easy interfacing to the object world, and the possibility to integrate data base systems.

\section{ARCHITECTURE FOR THE IMPLEMENTATION OF COOPERATIVE WORKSTATIONS}

Workstations with cooperative user interfaces must incorporate a variety of knowledge bases, data, and procedures that represent models, facts, and algorithms on quite different levels of abstraction. To handle the complexity of this situation for prototyping, a system structure has been designed (Fig 4) that separates (from bottom to top) between three layers.

1. The real-time layer includes workstation hardware and low-level (hardware-related) software. This layer has to offer solutions for all speed problems induced by high-resolution image storage, transmission, display, and processing operations. Although separated for speed reasons, the hardware and firmware are logically considered part of the object layer; they can be looked at as object methods implemented by means of separate high-speed processors.

2. The object layer is the object world with a complex structure of object classes, properties, and methods. Besides the objects of radiological interest, it also includes objects for interaction and user guidance (eg, menu fields and all kind of graphical objects).

3. The model layer includes the rule-based models of radiological information requirements in the context of tasks, users, and patient cases. In our demonstration system, the model layer acts as an intelligent consulting system that serves explicit re- 


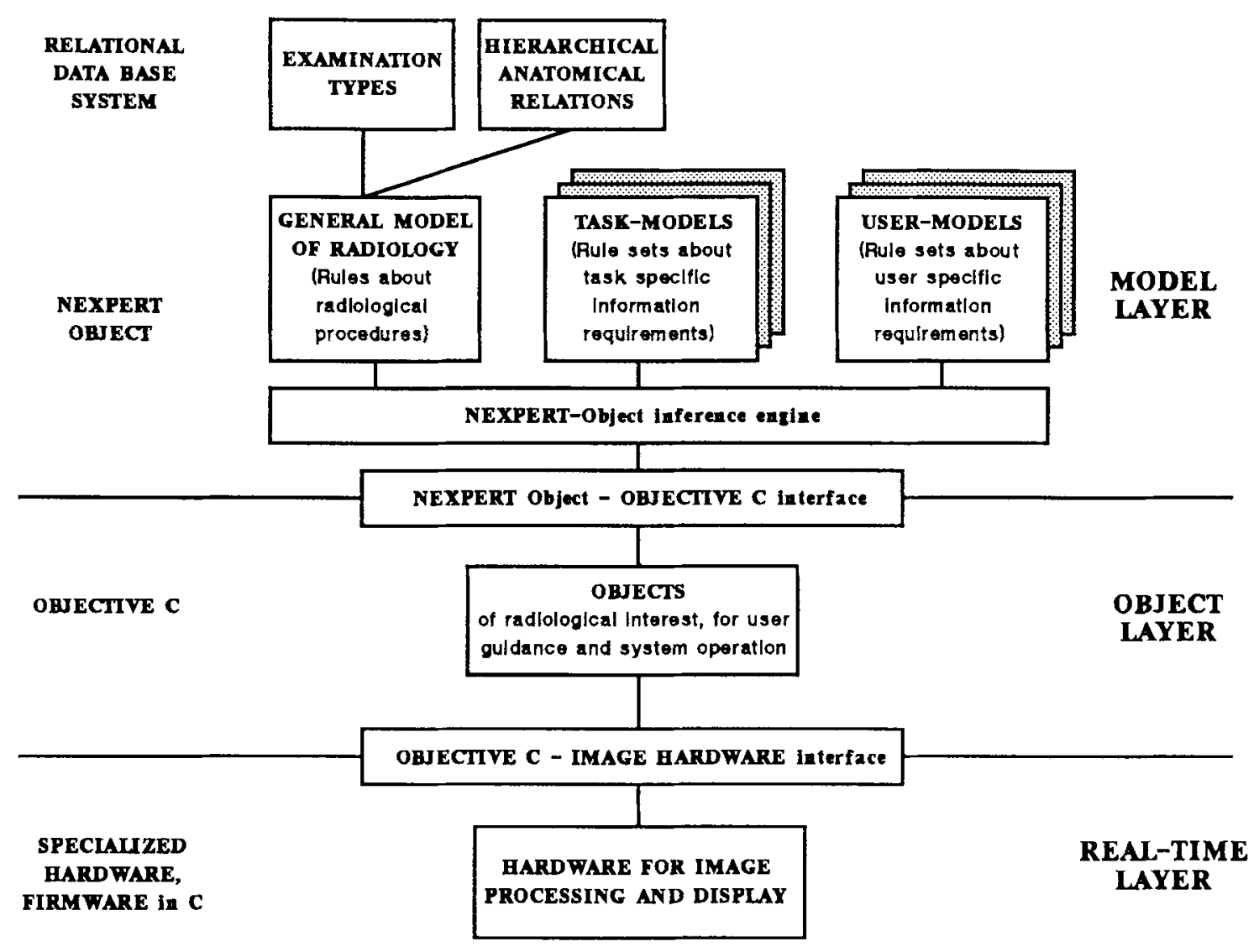

Fig 4. Block diagram of the layered workstation architecture.

quests initiated by objects (eg, the folder object may request a list of relevant images to be presented in a pictorial index). The model layer may also act on self-initiative by regularly scanning the properties of available object instances, deriving conclusions, and initiating actions in the object layer.

For demonstration of the adaptation mechanisms, all three system layers were realized on conventional off-the-shelf workstations (running both on VAX/VMS [Digital Equipment Corporation, Maynard, MA] and on Sun/UNIX workstations [Sun Microsystems, Inc, Mountain View, CA]).

\section{DISCUSSION AND PERSPECTIVE FOR FUTURE WORK}

An adaptive image workstation has been conceived that can act as a cooperative dialogue partner to support a number of tasks in diagnostic radiology, and a prototype has been made.
The cooperative user interface was designed to simplify and speed up image access and presentation in the technical environment of digital pictorial information systems in hospitals. The basic mechanism to achieve this was the automatic, context-dependent selection of images and their appropriate display arrangement. The resulting workstation prototype was demonstrated to a number of radiologists in a laboratory environment using real and complete patient cases. As a general comment, the surprisingly simple and convenient means of interaction was appreciated. In conclusion, there is a clear indication that the principle of modelbased cooperation offers the potential for better user interfaces in radiology by increasing the usability for various users and tasks. From the users' point of view, cooperative interfaces increase the efficiency of diagnostic work by apparently saving time and effort.

The realization of a cooperative workstation was possible only with a deep understanding of 
the clinical diagnostic process and the users' goal-oriented tasks in that process. The continuous involvement of end users was mandatory for the design process of the interface. As an advantage compared with nonadaptive interfaces, models of diagnostic information requirements not only implicitly guide the implementation, but are integral parts of the workstation's functionality. Namely the expliciteness and formality of models support the convenient and fast revision of design assumptions and facilitate the correction of misconceptions in prototyping. In summary, the design of model-based cooperative user interfaces appears as an effective technique in user-centered design.

In the course of the project, a first attempt was made to acquire and formalize models of diagnostic information requirements and to implement and integrate these models into a layered architecture of an adaptive image workstation. The hybrid software architecture using object-oriented and rule-based techniques offered a sound approach for knowledge representation and system integration. For the real-time layer, an appropriate hardware environment for image handling, processing, and display must be added for clinical evaluation.

Difficulties were identified in the insufficient clinical availability of a number of object properties needed for decision making. Some properties are not automatically available or, if available, not sufficiently formalized and standardized (eg, unified codes for examination types or the geometry of patient orientation, anatomic image descriptions, categories of clin- ical questions). In parts, this information must be delivered by radiology information system or hospital information service connections via standard interfaces. Standardization activities are required for a general applicability of the described model component.

The cooperative user interface design presented here should be regarded as a starting point. The models of diagnostic information requirements acquired so far certainly are incomplete and probably in parts are not precise enough. Furthermore, the variation of requirements among hospitals and different countries has not been considered sufficiently. Clear perspectives are indicated for follow-up activities on model validation and evaluation of the adaptation mechanism with cooperative image workstation prototypes in a clinical environment.

\section{ACKNOWLEDGMENT}

The acquisition of diagnostic information requirement models and their conversion to an object-oriented and rule-based representation system would have been impossible without the fruitful collaboration between the authors and the involved clinical partners of the University Hospitals of Aachen and Brussels. In particular and in the first place, we thank Dr Berthold Wein from the Clinic of Diagnostic Radiology in Aachen for his indispensible contributions in generating, structuring, and validating rules and in providing complete and detailed information about radiological work procedures, examinations, anatomic codes, and numerous other subjects that proved to be relevant for the project. We also thank S. Kaviani, who has provided a large number of protocols from routine viewing sessions taken at various workplaces in Aachen. From Brussels we appreciate the helpful input of Renaat van den Broeck, who gave valuable information in the initial knowledge acquisition phase.

\section{REFERENCES}

1. Norman DA: Cognitive engineering, in Norman DA Draper S (eds): User Centered System Design: New Perspectives in Human-Computer Interaction. Hillsdale, NJ, Erlbaum. 1986, pp 31-61

2. Braudes RE, Mun SK, Sibert J, et al: Workstation modelling and development: Clinical definition of a PACS user interface. Proc Med Imaging III, SPIE Vol 1093, 1989, pp 376-386

3. Wendler T, Wein B: A Rule-Based Model of Diagnostic Information Requirements for the Design of Adaptive Image Workstations. Proc CAR '91, Computer Assisted Radiology. Berlin, Germany, Springer, 1991, pp 629-635

4. Wendler T: Cooperative Human-machine Interfaces for Medical Image Workstations: A Scenario. Proc CAR'89,
Computer Assisted Radiology. Berlin, Germany, Springer, 1989, pp 775-779

5. Cox BJ: Object-oriented programming-An evolutionary approach. Addison Wesley, Reading, MA, 1987

6. Grewer R, Mönnich KJ, Schmidt J, et al: ObjectOriented Design for Medical Image Workstations. Proc CAR'89, Computer Assisted Radiology. Berlin, Germany, Springer, 1989, pp 780-784

7. Wendler T, Mönnich KJ, Schmidt J: Digital Image Workstations, in Osteaux $\mathrm{M}$ (ed): Hospital Integrated Picture Archiving and Communication Systems-A Second Generation PACS Concept. Berlin, Germany, Springer 1992, pp 73-210 